\title{
Experimental local realism tests without fair sampling assumption
}

\author{
G. Brida, M. Genovese, F.Piacentini \\ 1 I.N.RI.M., Strada delle Cacce 91, 10135 Torino, Italia \\ 2 e-mail: genovese@inrim.it
}

the date of receipt and acceptance should be inserted later

\begin{abstract}
Following the theoretical suggestion of Ref. 1,2, we present experimental results addressed to test restricted families of local realistic models, but without relying on the fair sampling assumption.

PACS. 03.65.Ta Foundations of quantum mechanics; measurement theory - 42.50.Xa Optical tests of quantum theory - 03.67.a Quantum information
\end{abstract}

Key words. QICS code: 02.30.Lh, Loopholes in Bell-type experiments

\section{Introduction}

The quest for a conclusive test of local realism has now a pluridecennial history 3, beginning with the celebrated 1965 Bell's paper 4 demonstrating that every Local Hidden Variable Theory (LHVT) cannot reproduce all the results of quantum mechanics when correlation among measurements on entangled states are considered. In particular, some inequalities always satisfied by every LHVT can be violated by Standard Quantum Mechanics (SQM).

Since then many experiments have been realized for testing Bell inequalities [3], with a recent intensification due to the application to the emerging field of quantum information. Nevertheless, whilst a clear spacelike separation has been unequivocally achieved, still a conclusive test is missing, because of low detection efficiency that requires the additional hypothesis that the observed sample is a faithful representation of the whole one (the so-called fair sampling assumption, leading to the detection loophole).

In the last years relevant progresses for eliminating this loophole have been realized [3], both based on improvements of detectors and on the result that for nonmaximally entangled states the limit for an elimination of this problem is lowered from $82 \%$ to $66.7 \%$ [5,6]. However, a conclusive experiment is still missing.

On the other hand, specific local realistic models built for surviving traditional Bell inequalities tests, as stochastic optics [7] (a branch of stochastic elecrodynamics [8]), have been falsified by specifically addressed experimental tests $3,9,10$.

Recently, some works appeared [1,2] with the purpose to propose some specific inequality verified by a restricted families of LHVT, but whose test, realizable by exploiting polarization entangled photon pairs generated by parametric down-conversion (PDC), would be free from the detection loophole. The aim of this paper is to present an experimental test of these theoretical proposals.

\section{Theoretical motivations}

In 1,2 a set-up based on polarization bipartite entangled states of photons is considered, where one measures the single count rates $R_{1}$ and $R_{2}$ on the idler and signal arm, the coincidence rate $R_{12}$ and the total pair production rate $R_{0}$. One has

$$
R_{1}=R_{2}=\frac{1}{2} \eta R_{0} \quad R_{12}=\frac{1}{4} \eta^{2} R_{0}[1+V \cos (2 \phi)]
$$

where $\phi$ is the angle between the two polarization axes, $V$ the observed visibility and $\eta$ the detection efficiency (considered identical for the two arms).

The results of such an experiment can be reproduced by a LHV model constituted by the functions [1]:

$$
\begin{array}{r}
\rho\left(\left|\lambda_{1}-\lambda_{2}\right|\right) \geq 0 \quad 0 \leq P_{j}\left(\left|\lambda_{j}-\phi_{j}\right|\right) \leq 1 \quad j=1,2 \\
\int \rho\left(\left|\lambda_{1}-\lambda_{2}\right|\right) d \lambda_{1} d \lambda_{2}=1
\end{array}
$$

where $\lambda_{1}, \lambda_{2}$ are the hidden (angular) variables and $\phi_{1}, \phi_{2}$ the angles of the polarization planes with respect to an axis perpendicular to pump direction and polarization. The predictions for the single count and coincidence rates are:

$$
\begin{array}{r}
\frac{R_{j}}{R_{0}}=\int \rho\left(\left|\lambda_{1}-\lambda_{2}\right|\right) P_{j}\left(\left|\lambda_{j}-\phi_{j}\right|\right) d \lambda_{1} d \lambda_{2} \\
\frac{R_{12}}{R_{0}}=\int \rho\left(\left|\lambda_{1}-\lambda_{2}\right|\right) \prod_{j=1}^{2} P_{j}\left(\left|\lambda_{j}-\phi_{j}\right|\right) d \lambda_{1} d \lambda_{2}
\end{array}
$$


From this model, introducing the visibility parameters:

$$
\begin{gathered}
V_{A}=\frac{R_{12}(0)-R_{12}(\pi / 2)}{R_{12}(0)+R_{12}(\pi / 2)} \\
V_{B}=\sqrt{2} \frac{R_{12}(\pi / 8)-R_{12}(3 \pi / 8)}{R_{12}(\pi / 8)+R_{12}(3 \pi / 8)}
\end{gathered}
$$

one derives the inequality [1]:

$$
\frac{V_{B}}{V_{A}} \geq F=1+\cos ^{2}\left(\frac{\pi \eta}{2}\right)\left[V_{B}-\frac{\sin ^{2}(\pi \eta / 2)}{(\pi \eta / 2)^{2}}\right]
$$

that can be violated in quantum mechanics (where, in the ideal case, $\left.V_{B} / V_{A}=1\right)$. An accurate test of this inequality will be the first achievement of our experiment [11.

Then we consider another inequality of the same type, but valid for a larger family of LHVT [2. In this case, one takes into account a set of four hidden variables $\lambda \equiv$ $\left\{\chi_{1}, \chi_{2}, \mu_{1}, \mu_{2}\right\}$ (of two different types) instead of two. The equations of this local realistic model are [2]:

$$
\begin{gathered}
\rho(\lambda)=\rho_{\chi}\left(\left|\chi_{1}-\chi_{2}\right|\right) g_{1}\left(\mu_{1}\right) g_{2}\left(\mu_{2}\right) \geq 0 \\
\int \rho_{\chi}\left(\left|\chi_{1}-\chi_{2}\right|\right) d \chi_{1} d \chi_{2}=\int g_{j}\left(\mu_{j}\right) d \mu_{j}=1 \\
0 \leq P_{j}\left(\left|\chi_{j}-\phi_{j}\right|\right)=\int Q_{j}\left(\mu_{j},\left|\chi_{j}-\phi_{j}\right|\right) g_{j}\left(\mu_{j}\right) d \mu_{j} \leq 1
\end{gathered}
$$

and give the expected ratios (after integrating over the $\mu_{j}$ variables):

$$
\begin{array}{r}
\frac{R_{j}}{R_{0}}=\int \rho_{\chi}\left(\left|\chi_{1}-\chi_{2}\right|\right) P_{j}\left(\left|\chi_{j}-\phi_{j}\right|\right) d \chi_{1} d \chi_{2} \\
\frac{R_{12}}{R_{0}}=\int \rho_{\chi}\left(\left|\chi_{1}-\chi_{2}\right|\right) \prod_{j=1}^{2} P_{j}\left(\left|\chi_{j}-\phi_{j}\right|\right) d \chi_{1} d \chi_{2}
\end{array}
$$

Let us consider a system with rotational invariance, in which the coincidence rates depend only on the relative polarization orientation $\phi=\phi_{1}-\phi_{2}$, and put the probabilities $P_{1}=P_{2}=P$ (perfect symmetry between the apparatuses on the two branches of PDC). By introducing the parameter (where ()$_{+}$means putting zero if the quantity inside the bracket is negative):

$$
D(\eta) \equiv \frac{4}{3 \pi} \sqrt{\frac{2}{3 \eta}-\frac{1}{2}-\frac{\sin ^{4}(\pi \eta / 2)}{(\pi \eta / 2)^{4}}}\left(V-\frac{\sin ^{2}(\pi \eta / 2)}{(\pi \eta / 2)^{2}}\right)_{+}^{\frac{3}{2}}
$$

with:

$$
V=2 \frac{\sum_{j=1}^{n} R_{12}\left(\phi_{j}\right) \cos \left(2 \phi_{j}\right)}{\sum_{j=1}^{n} R_{12}\left(\phi_{j}\right)}
$$

one derives the following inequality:

$$
\Delta_{\text {min }}=\left[\frac{n \sum_{j=1}^{n} R_{12}^{2}\left(\phi_{j}\right)}{\left(\sum_{j=1}^{n} R_{12}\left(\phi_{j}\right)\right)^{2}}-\right.
$$

$$
\left.-2 \frac{\left(\sum_{j=1}^{n} R_{12}\left(\phi_{j}\right) \cos \left(2 \phi_{j}\right)\right)^{2}}{\left(\sum_{j=1}^{n} R_{12}\left(\phi_{j}\right)\right)^{2}}-1\right]^{(1 / 2)} \geq D(\eta)
$$

where $\phi_{j}=\pi \cdot(j-1) / n$.

Testing this second inequality will be the second purpose of the present work.

\section{Experimental set-up and results}

In order to test these inequalities, we measured the variation of the coincidence rate $R_{12}(\phi)$ with respect to the variable $\phi$, using the experimental setup reported in Fig. 1 .

In our set-up a $0.2 \mathrm{~W}, 398 \mathrm{~nm}$ pulsed (with $200 \mathrm{fs}$ pulses) laser beam, obtained by second harmonic generation from a titanium-sapphire beam at $796 \mathrm{~nm}$, pumps a type-II BBO 5x5x0,5 mm crystal. With a proper selection of the phase-matching conditions, we obtain the collinear degenerate $(796 \mathrm{~nm}) \mathrm{PDC}$ and send it to a non-polarizing 50\%-50\% beam splitter (BS). Passing through the BS, the state changes like this:

$$
|H V\rangle \rightarrow \frac{1}{2}\left(\left|H_{r} V_{r}\right\rangle+\left|H_{t} V_{t}\right\rangle+\left|V_{r} H_{t}\right\rangle+\left|H_{r} V_{t}\right\rangle\right)
$$

where the pedices $r$ and $t$ respectively indicate the reflection and transmission directions.

By postselecting the cases when photons follow different paths, one obtains the Bell state:

$$
\left|\psi_{+}\right\rangle=\frac{1}{\sqrt{2}}\left(\left|V_{r} H_{t}\right\rangle+\left|H_{r} V_{t}\right\rangle\right)
$$

On the transmission $(t)$ arm we put a Glan-Thomson polarizer, a pinhole, an interference filter (FWHM $10 \mathrm{~nm}$, $\lambda_{\text {peak }} 795 \mathrm{~nm}$ ) and an optical fiber coupler connected with a multi-mode fiber to the detector, a Perkin-Elmer SPCMAQR-14-FC-9491 silicon avalanche photo-diode (quantum efficiency at $796 \mathrm{~nm}: 62 \%$ ); the reflection $(r)$ arm featured a Glan-Thomson polarizer, a large band interference filter (FWHM $40 \mathrm{~nm}, \lambda_{\text {peak }} 800 \mathrm{~nm}$ ) and the same fiber coupling and detection devices as in the other arm. Taking the $t$ photons as trigger, a coincidence window is opened on the arm $r$ detector to reveal the correlated pairs: this is obtained by sending the output of the $t$ detector as start to a Time-to-Amplitude Converter (TAC) that receives the $r$ detector signal as stop. The $20 \mathrm{~ns}$ coincidence window is set properly to exclude spurious coincidences with PDC photons of the following pulse (we remind that the repetition rate of the laser is $70 \mathrm{MHz}$ ). The TAC output is then acquired via PC, together with the TAC's Valid Start counts (giving us the total number of opened coincidence windows). The background has been evaluated and subtracted by measuring the TAC output of the system without PDC generation (simply rotating the pump polarization by 90 before the nonlinear crystal).

To violate the inequalities presented in the previous section, we fixed the polarization axis of both the GlanThomson polarizers at an angle of $\frac{\pi}{4}$ with the horizontal 
plane, and then we performed a scan of the coincidence rates $R(\phi)$ (where $\phi$ represents the difference between the polarization planes) simply rotating the polarizer on the $r$ arm by $\frac{\pi}{8}$ per step, until we cover the whole interval $\phi \in[0, \pi[:$ the rate obtained for each step is the average of 30 different acquisitions of $30 \mathrm{~s}$ each.

The obtained curve is shown in fig. 2 .

As a first test for our entangled state, we checked (of course within the fair sampling hypothesis) the ClauserHorne inequality [3], written in the form:

$$
\frac{\left|R\left(\frac{\pi}{8}\right)-R\left(\frac{3 \pi}{8}\right)\right|}{R_{\text {tot }}} \leq \frac{1}{4}
$$

The collected data, with a measured visibility $97.8 \%$, led to the following violation:

\begin{tabular}{|c|c|c|}
\hline CH violation & $\sigma$ & $\frac{\text { violation }}{\sigma}$ \\
\hline 0,1026 & 0,0021 & 47,8 \\
\hline & \multicolumn{2}{|l}{} \\
\hline
\end{tabular}

Then, to violate the inequality 6 , we evaluated the quantities:

\begin{tabular}{|c|c|}
\hline$V_{B}$ & $V_{A}$ \\
\hline $0,9985 \pm 0,0030$ & $0,9784 \pm 0,0017$ \\
\hline \hline$V_{B} / V_{A}$ & $F$ \\
\hline $1,0205 \pm 0,0048$ & $1,0876 \pm 0,0009$ \\
\hline & \multicolumn{1}{|c}{ where, following the } \\
\hline
\end{tabular}

indications of Ref. [2, we used for the value of the quantum efficiency the nominal detector efficiency as reported on the manufacturer data sheet for our wave length, $\eta=$ $62 \%$.

Of course, the difference of $V_{A}, V_{B}$ from unit and from each other derives from unavoidable imperfections of the experimental apparatus; nevertheless a clear violation of the inequality 6 is observed:

\begin{tabular}{|c|c|c|}
\hline$F-V_{B} / V_{A}$ & $\sigma$ & $\frac{\text { violation }}{\sigma}$ \\
\hline 0,0671 & 0,0057 & 11,7 \\
\hline & \multicolumn{3}{|l}{} \\
\hline
\end{tabular}

Finally, we present the results for the inequality (2) (with again $\eta=62 \%$ ). Our data led to a violation:

\begin{tabular}{|c|c|c|}
\hline$D(\eta)-\Delta_{\min }$ & $\sigma$ & $\frac{\text { violation }}{\sigma}$ \\
\hline 0,0073 & 0,0022 & 3,3 \\
\hline & \multicolumn{2}{|l}{} \\
\hline
\end{tabular}

For the sake of completeness, we mention that both the inequalities (62) still are violated even without background subtraction (of 11.6 and 1.7 standard deviations respectively).

Thus, we have clearly shown a violation of the predictions made upon the LHVT models presented in [1] and [2, whilst perfectly agreeing with SQM.

\section{Conclusions}

In summary, we have experimentally demonstrated, following the theoretical predictions of [1,2], that the specific families of hidden variable theories considered there fail to properly reproduce the observed correlations between measurements of bipartite polarization photon entangled states, and are therefore falsified by our experiment.

This work has been supported by MIUR (PRIN 2005023443002), by Regione Piemonte (E14) and by grant RFBR-Piedmont 07-02-91581-ASP.

We acknowledge fruitful discussions with E. Santos.

\section{References}

1. E. Santos, Phys. Lett. A 327 (2004) 33.

2. E. Santos, quant-ph0612212 Eur. Phys. Journ D in press.

3. M. Genovese, Phys. Rep. 413 (2005) 319.

4. J.S. Bell, Physics 1 (1965) 195.

5. P. H. Eberhard, Phys. Rev. A 47, R747 (1993).

6. G. Brida et al., Phys. Lett. A 268 (2000) 12.

7. A. Casado et al., J. Opt. Soc. Of Am. B 14 (1997) 494; A. Casado et al., Phys. Rev. A 55 (1997) 3879. A. Casado et al., Phys. Rev. A 56 (1997) 2477. A. Casado et al., J. Opt. Soc. Of Am. B 15 (1998) 1572; A. Casado et al., Eur. Phys. Journ. D 11 (2000) 465; D 13 (2001) 109; A. Casado et al., quant-ph 0202097. K. Dechoum, T.W.Marshall and E.Santos, Journ. Of Mod. Opt. 47 (2000) 1273; T.W. Marshall, quant-ph 0203042 .

8. T.W. Marshall, Proc. Cambridge Philos. Soc. 61, 537 (1965); T. H. Boyer, Phys. Rev. 182, 1374 (1969); E. Santos, Lett. Nuovo Cimento 4, 497 (1972); M. Surdin, Ann. Inst. Henri Poincaré 15, 203 (1971); L. de la Pena and A.M. Cetto, "The quantum dice : and introduction to stochastic electrodynamics", Kluwer, 2000.

9. G. Brida et al., Phys. Lett. A 299 (2002) 121. G. Brida et al., Journ. Mod. Opt. 11 (2003) 1757.

10. G. Brida et al., quant-ph/0612075 Journ. of Phys. Conf Ser. in press.

11. M. Genovese et al., Opt. and Spec. 99 (2005) 185. 


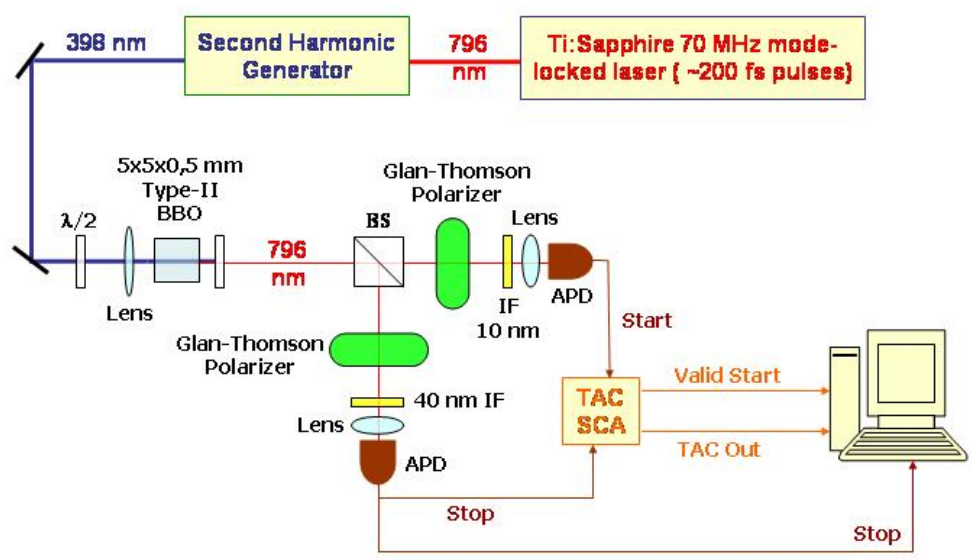

Fig. 1. Scheme of the experimental setup.

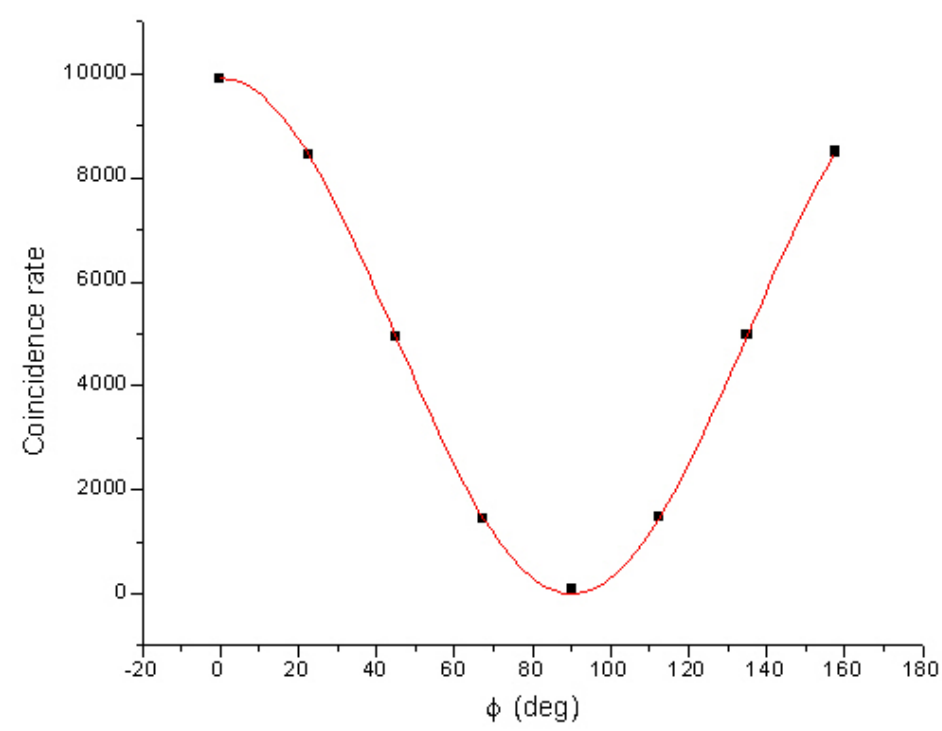

Fig. 2. Coincidence rates versus $\phi$ (angle between the axes of $t$ and $r$ polarizers). The experimental data are compared with the theoretical curve $\cos ^{2}(\phi) / 2$, with whom are in perfect agreement (correlation coefficient $r=0.9998)$. Uncertainty bars are too small for being visible. 